\title{
Deformation properties of the Barcelona-Catania-Paris (BCP) energy density functional
}

\author{
L. M. Robledo* \\ Departamento de Física Teórica C-XI, Universidad Autónoma de Madrid, E-28049 Madrid, Spain \\ M. Baldo \\ Instituto Nazionale di Fisica Nucleare, Sezione di Catania, Via Santa Sofia 64, I-95123 Catania, Italy \\ P. Schuck \\ Institut de Physique Nuclèaire, IN2P3-CNRS, UMR8608, F-91406 Orsay, France and \\ Université Paris-Sud, F-91406 Orsay, France
}

X. Viñas

Departament d'Estructura i Constituents de la Matèria and Institut de Ciències del Cosmos, Universitat de Barcelona, Diagonal 647, E-08028 Barcelona, Spain

(Received 24 January 2008; published 12 May 2008)

\begin{abstract}
We explore the deformation properties of the newly postulated Barcelona-Catania-Paris (BCP) energy density functional (EDF). The results obtained for three isotope chains of $\mathrm{Mg}$, Dy, and $\mathrm{Ra}$ are compared to the available experimental data as well as to the results of the Gogny-D1S force. Results for the fission barrier of ${ }^{240} \mathrm{Pu}$ are also discussed.
\end{abstract}

DOI: 10.1103/PhysRevC.77.051301

PACS number(s): 21.60.Jz, 21.10.Dr, 21.30.-x

In a recent work [1] we have shown that a fully microscopic input from nuclear and neutron equation of state (EOS) calculations [2] complemented by additional terms accounting for finite size effects, to nuclear density functional theory (DFT) can be very successful. The density functional for the ground state energy $E$ of a nucleus, we considered in [1], is of the form

$$
E=T_{0}+E^{s . o .}+E_{\mathrm{int}}^{\infty}+E_{\mathrm{int}}^{F R}+E_{C},
$$

where $T_{0}$, E $E^{\text {s.o. }}, E_{C}$ are the standard expressions for kinetic energy, spin-orbit term, and Coulomb energy. The dependence on proton and neutron densities in $E_{\text {int }}^{\infty}$ is the one obtained from the microscopic calculation [2] and treated in the local density approximation. We added a finite range (FR) term to account for a more physical, i.e., wider density profile and, therefore, a more correct value of the surface energy

$$
\begin{aligned}
E_{\mathrm{int}}^{F R}\left[\rho_{n}, \rho_{p}\right]= & \frac{1}{2} \sum_{t, t^{\prime}} \iint d^{3} r d^{3} r^{\prime} \rho_{t}(\mathbf{r}) v_{t, t^{\prime}}\left(\mathbf{r}-\mathbf{r}^{\prime}\right) \rho_{t^{\prime}}\left(\mathbf{r}^{\prime}\right) \\
& -\frac{1}{2} \sum_{t, t^{\prime}} \gamma_{t, t^{\prime}} \int d^{3} r \rho_{t}(\mathbf{r}) \rho_{t^{\prime}}(\mathbf{r})
\end{aligned}
$$

with $t=$ proton/neutron and $\gamma_{t, t^{\prime}}$ the volume integral of $v_{t, t^{\prime}}(r)$. The second term in the right-hand side (r.h.s.) of Eq. (2) is introduced to preserve the nuclear matter properties of the microscopic calculation in the bulk. For the finite range form factor $v_{t, t^{\prime}}(r)$ we made a simple Gaussian ansatz, that is $v_{t, t^{\prime}}=V_{t, t^{\prime}} e^{-r^{2} / r_{0}^{2}}$. We chose a minimum of three open parameters: $V_{p, p}=V_{n, n}=V_{L}, V_{n, p}=V_{p, n}=V_{U}$, and $r_{0}$. The values of these parameters together with the spin-orbit

\footnotetext{
*1uis.robledo@uam.es
}

strength can be found in [1]. For the pairing part of the interaction we simply take the density dependent delta force studied in [3] for an effective mass equal to the bare one. The parameters $V_{L}, V_{U}$, and $r_{0}$ as well as the spin-orbit strength $W_{0}$ were adjusted in the usual way to reproduce the ground state energy of only some selected spherical nuclei (BCP1) and, additionally, some experimental charge radii (BCP2). With this so-called Barcelona-Catania-Paris (BCP) functional excellent results for 161 even-even spherical nuclei with rms values for ground state energies and charge radii, comparable with the most performing functionals on the market [4-7], were obtained.

In this Rapid Communication we continue investigating the properties of the BCP functional and explore how deformation properties of nuclei are described. We will find that the performance is again excellent, as well in comparison with experiment as in comparison with the results of the very successful Gogny D1S force [4]. We have computed the potential energy surfaces (PES) as a function of the axially symmetric quadrupole deformation of several isotopes of the magnesium, dysprosium, and radium species with the idea in mind of covering different regions and different nuclear scenarios of the nuclide chart. To finish these exploratory calculations we have considered also the fission barrier of the heavy nucleus ${ }^{240} \mathrm{Pu}$ which is very sensitive to quadrupole deformation properties.

As it is customary in this kind of calculations the HFB equation has been recast as a minimization process of the energy density functional where the HFB wave function of the Bogoliubov transformation [8] is chosen to minimize the energy. The variational set of HFB wave functions is given by means of the standard Thouless parametrization [8]. The minimization process is performed by using the gradient method as it allows an easy and efficient implementation of 
constraints by using the technique of Lagrange multipliers. For the present exploratory calculations we have restricted the calculation to configurations preserving axial symmetry (but reflection symmetry breaking is allowed) in order to reduce the computational effort. The quasiparticle operators are expanded in a harmonic oscillator basis written as the tensor product of one oscillator in the $z$ direction with oscillator frequency $\omega_{z}$ and another in the perpendicular one and characterized by $\omega_{\perp}$. Large enough basis (up to 26 shells in the $z$ direction in the case of fission) and optimization of the harmonic oscillator lengths have been used to guarantee good convergence for the values of all physical quantities.

In the calculations with the Gogny force the standard approximations have been considered, namely the Coulomb exchange contribution to the energy has been replaced by the Slater approximation and the Coulomb pairing field has been neglected. The two body kinetic energy correction has been fully taken into account. For details pertaining the implementation of the HFB procedure and center of mass correction with the BCP functional, see Ref. [1].

We have performed calculations of the potential energy surface (PES), as a function of the mass quadrupole moment $Q_{20}$, of several isotopes of the nuclei of magnesium, dysprosium, and radium. The idea is to explore some representative nuclei distributed all over the nuclide chart. We have compared the results of the calculations with the BCP energy density functional (parametrizations $\mathrm{BCP} 1$ and $\mathrm{BCP} 2$ ) with those obtained by using the D1S Gogny force which is considered here as a benchmark. For the $\mathrm{Mg}$ isotopes we have computed the even mass ones between $A=20$ and $A=40$ (covering the $N=8$ and $N=20$ neutron shell closures). For the dysprosium isotopes we have computed the ones from $A=140(N=74)$ up to $A=170(N=104)$. Finally, for the radium isotopes we have considered isotopes between $A=216(N=128)$ up to $A=236(N=148)$. In Fig. 1 we have plotted the PES (as a function of the $\beta_{2}$ deformation parameter, defined as $\beta_{2}=\sqrt{\frac{4 \pi}{5}} \frac{Q_{20}}{\left\langle r^{2}\right\rangle}$ ) of some representative nuclei. As the results obtained with $\mathrm{BCP} 1$ and $\mathrm{BCP} 2$ parametrizations are almost identical, only the first ones are shown. The first noticeable fact is that in all the nuclei studied the two curves look rather similar showing minima, maxima, and saddle points almost at the same $\beta_{2}$ values. However, the relative energy of those configurations obtained with $\mathrm{BCP} 1$ is different from the one with the Gogny force. According to the number of neutrons we can distinguish two different regions: the first one corresponds to neutron numbers greater than the midshell value, where there are differences in the prolate side between Gogny and BCP which extend up to rather high $\beta_{2}$ values of around 0.5 . Examples of this behavior are the nuclei ${ }^{26} \mathrm{Mg}$ $(N=14),{ }^{32} \mathrm{Mg}(N=20)$, and ${ }^{144} \mathrm{Dy}(N=78)$. The second region corresponds to neutron numbers lower than the midshell value where the spherical configuration lies, in the Gogny case, at an energy higher than in the BCP results and the difference increases as $N$ increases. This means that the deformation energy $E_{\text {def }}$, defined as the energy difference between the spherical configuration and the deformed ground state, is larger for the Gogny force than for the BCP functional. As a consequence of this behavior the excitation energy of the
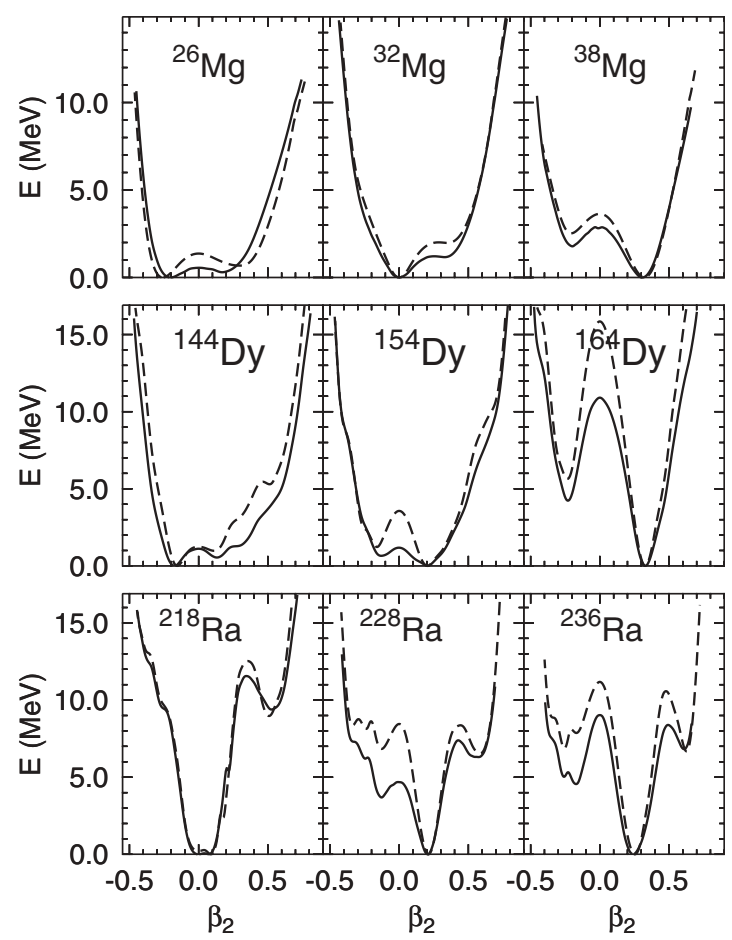

FIG. 1. The potential energy surfaces (PES) of some representative nuclei as a function of the $\beta_{2}$ deformation parameter (full line, $\mathrm{BCP} 1$ functional; dashed one Gogny-D1S force). In all cases the zero of the curves refers to their absolute minimum.

oblate minimum is higher with the Gogny interaction than with the BCP functional, reaching the difference roughly a factor of two, as in the example shown of ${ }^{228} \mathrm{Ra}$. Examples of this behavior shown in Fig. 1 are ${ }^{154} \mathrm{Dy},{ }^{164} \mathrm{Dy},{ }^{228} \mathrm{Ra}$, and ${ }^{236} \mathrm{Ra}$. Finally, for some nuclei close or at shell closure like ${ }^{218} \mathrm{Ra}$ shown in Fig. 1 the two PES are rather similar. These systematic differences observed between Gogny and BCP1 could be a consequence of the different surface energy coefficient $a_{S}$. Its value is pretty similar for BCP1 and BCP2, $a_{S}=17.74 \mathrm{MeV}$ and $17.84 \mathrm{MeV}$, respectively, and differ by $2 \%$ from the Gogny D1S value of $a_{S}=18.2 \mathrm{MeV}$ [9]. This minute difference could be enough to explain the observed differences (see [5] for a study of its effect on fission barriers). It has to be mentioned that the values given here for $a_{S}$ were computed including the spin-orbit interaction and therefore the Gogny D1S value is $0.8 \mathrm{MeV}$ lower than the value given in [5]. Another possible explanation for the differences in the excitation energy of prolate and oblate minima could be the lower level density obtained with the Gogny force (effective mass ratio of 0.7 ) as compared with the one of the $\mathrm{BCP}$ functional (effective mass equal to the physical one) that makes shell gaps stronger (see below). The deformation energy $E_{\text {def }}$ also depends on the amount of pairing correlations (see, for example, Ref. [10]) in such a way that the stronger the pairing correlations are the smaller the value of $E_{\text {def }}$. It turns out that the particle-particle correlation energy $E_{p p}=\operatorname{Tr}(\Delta \kappa)$, which is a measure of pairing correlations, is typically 30 to $40 \%$ stronger in the Gogny-D1S case. However, its effect on $E_{\mathrm{def}}$ is not strong enough as to overcome the other effects mentioned 
above that lead to an increase of the deformation energy of Gogny D1S with respect to BCP.

Concerning the physics, we observe in Fig. 1 how shape coexistence in ${ }^{26} \mathrm{Mg}$ with its oblate ground state appears in both the BCP and D1S calculations. We also observe the shoulder precursor of the deformed ground state after angular momentum projection in ${ }^{32} \mathrm{Mg}$ as well as the prolate ground state that develops in heavier than ${ }^{32} \mathrm{Mg}$ isotopes as a consequence of neutrons populating the $f p$ shell (see ${ }^{38} \mathrm{Mg}$ ). In the case of Dy we have two examples of shape coexistence with an oblate ground state $\left({ }^{144} \mathrm{Dy}\right)$ and a prolate one $\left({ }^{154} \mathrm{Dy}\right)$ as well as a well deformed system like ${ }^{164} \mathrm{Dy}$. Shape coexistence also appears in ${ }^{142} \mathrm{Dy}$ (not shown) but in this case BCP predicts a prolate g.s. whereas Gogny predicts an oblate one. Finally in the lower row of Fig. 1 we have several $\mathrm{Ra}$ isotopes ranging from the spherical ${ }^{218} \mathrm{Ra}$ showing an excited superdeformed minimum, to the reasonably well deformed ${ }^{228} \mathrm{Ra}$ and ${ }^{236} \mathrm{Ra}$ where the excitation energies of the oblate minima are quite a bit higher in the case of the Gogny force than with the BCP functional.

In Fig. 2 we have displayed the magnitude of several physical quantities corresponding to the ground state of the computed Dy isotopes, using the two BCP functionals and the D1S force. In the lower panel of this figure the two neutron separation energies are plotted. The typical discontinuity at

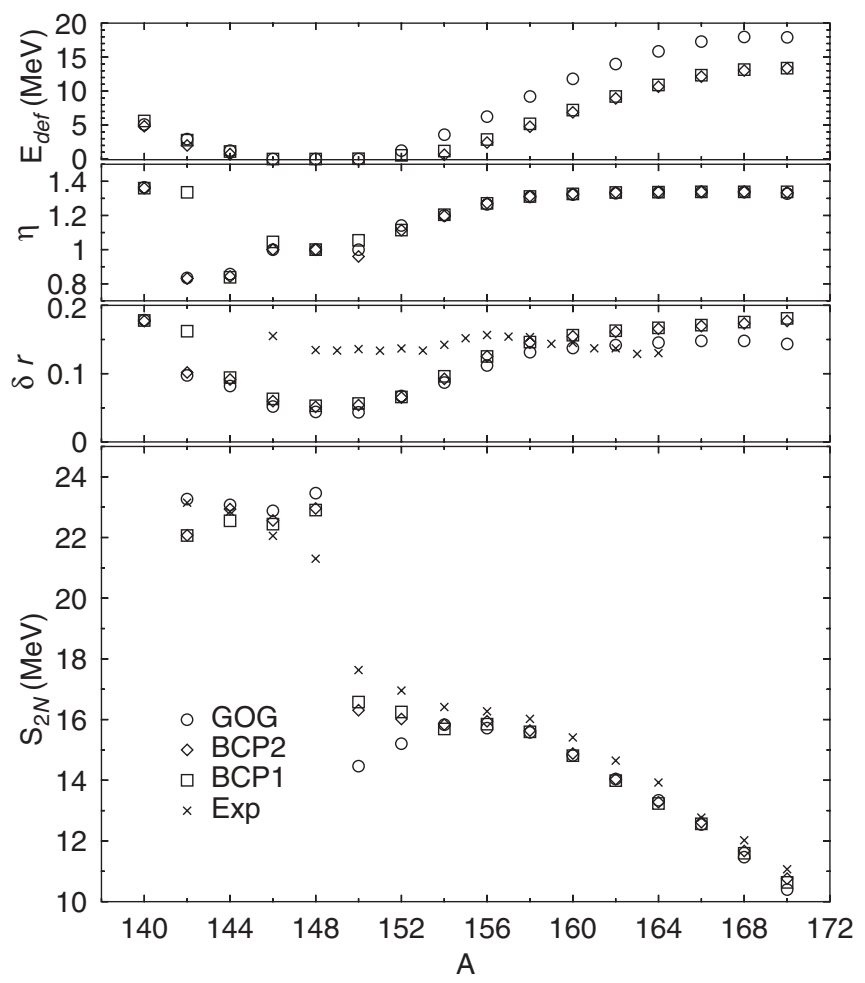

FIG. 2. Mean field results for the self-consistent minimum of all the Dy isotopes considered. In the lower panel the two neutron separation energy $S_{2 N}$ is shown against the mass number of the isotopes along with the experimental data. In the next panel the quantity $\delta r=\left\langle r^{2}\right\rangle^{1 / 2}-\sqrt{3 / 5} \times 1.2 \times A^{1 / 3}$ is depicted. In the following panel the axis ratio $\eta$ (see text for details) is represented. Finally, in the upper panel the deformation energy $E_{\text {def }}$ is plotted. the semimagic nucleus ${ }^{148}$ Dy $(N=82, Z=66)$ is clearly observed. As is usual with effective mean field approaches, our predictions do not compare well with experiment when close to a semimagic configuration. However, the agreement is much better for well-deformed systems. The absolute values of the binding energies agree well in all the cases and, as an example, we can mention that in a calculation with a basis of 15 shells the binding energies of ${ }^{160} \mathrm{Dy}$ are $-1305.894 \mathrm{MeV}$ for BCP1, - 1305.607 MeV for BCP2, -1304.288 MeV for $\mathrm{D} 1 \mathrm{~S}$ whereas the experimental value is $-1309.457 \mathrm{MeV}$. The theoretical results do not include any kind of correlation energy beyond mean-field like the rotational energy correction that can be estimated to be $2.18 \mathrm{MeV}$ for BCP and 3.16 MeV for D1S. In the next panel of Fig. 2 a quantity related to the mean square radius, namely $\delta r=\left\langle r^{2}\right\rangle^{1 / 2}-\sqrt{3 / 5} \times 1.2 \times A^{1 / 3}$, is plotted. We observe that the three theoretical predictions compare rather well with experimental data and surprisingly the radii are closer to experiment in well deformed nuclei which were not considered in the original fit [1]. The different result obtained in ${ }^{142}$ Dy for BCP1 and BCP2 (and D1S) is due to the almost degenerate oblate and prolate minima in this nucleus: with BCP1 the ground state is prolate whereas it is oblate with $\mathrm{BCP} 2$ and Gogny-D1S. In the next panel the axis ratio $\eta=\left(\left\langle z^{2}\right\rangle /\left\langle x^{2}\right\rangle\right)^{1 / 2}$ which is a measure of deformation $\left(\beta_{2}=\right.$ $\left.\sqrt{\frac{4 \pi}{5}} \frac{\eta^{2}-1}{\eta^{2}+2}\right)(\eta>1$ for prolate deformation, $\eta<1$ for oblate deformations and $\eta=1$ for spherical states) is plotted. As in previous cases, the agreement between the three theoretical results is very good confirming what was said in discussing Fig. 1 about the coincidence of the position of maxima and minima of the PES. Finally in the upper panel the deformation energy $E_{\text {def }}$ is shown. The Gogny-D1S values for this magnitude are systematically larger by a few $\mathrm{MeV}$ than the BCP ones as discussed previously.

The results for the self-consistent ground state in the $\mathrm{Mg}$ isotopes is depicted in Fig. 3 where we have only plotted the curves for $S_{2 N}$ and $\delta r$. The two additional quantities shown in the case of the Dy isotopes have a similar behavior for the $\mathrm{Mg}$ nuclei and are not presented here. Again, we observe for the $S_{2 N}$ a reasonably good agreement with experiment which is of the same quality for the three schemes. The theoretical radii

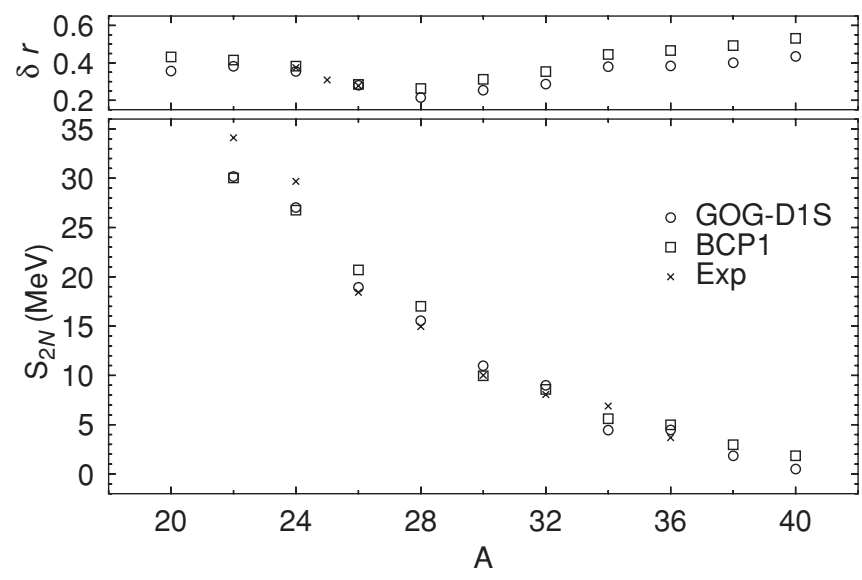

FIG. 3. Same as Fig. 2 but for the $\mathrm{Mg}$ isotopes and only the two lower panels. 


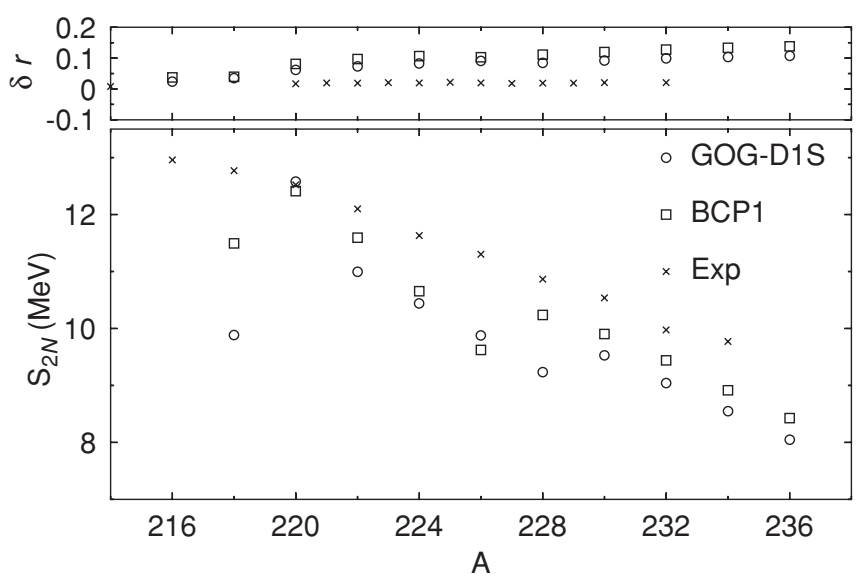

FIG. 4. Same as Fig. 3 but for the Ra isotopes.

also look rather similar in all the theoretical approaches and compare well with the scarce experimental values.

In Fig. 4 we show the self-consistent results for the $S_{2 N}$ of the $\mathrm{Ra}$ isotopes. The results for the isotopes ${ }^{220-226} \mathrm{Ra}$ include octupole deformation in their ground state with $\beta_{3}$ values of the order of 0.15 for all the cases considered. It is not the scope of the present work to discuss octupole deformation in detail but it is worth pointing out that also this deformation multipole comes out quite the same independent of the force or functional considered. The theoretical $S_{2 N}$ results look rather similar except for the nucleus ${ }^{218} \mathrm{Ra}$ which is close to the semimagic ${ }^{214} \mathrm{Ra}$. As it was discussed before, the two neutron separation energies have a somewhat erratic behavior around semimagic configurations. Most surprising is the region around $A=226-228$ where the theoretical predictions move away from experimental values. Concerning the radii a good agreement between the results obtained with $\mathrm{BCP}$, Gogny, and the experiment is observed.

In Fig. 5 we display the potential energy surface (PES) as a function of the quadrupole moment corresponding to the fission process of the nucleus ${ }^{240} \mathrm{Pu}$ computed using the two $\mathrm{BCP}$ functionals and the Gogny force. As it is customary in this kind of calculations [11] the rotational energy correction computed with the Yoccoz moment of inertia has been included in the PES. It can be concluded from this figure (lower panel) that the PES obtained with the BCP1 and BCP2 functionals are very similar and both closely follow the shape of the Gogny D1S PES up to the second minimum. The heights of the fission barriers provided by both the BCP1 and BCP2 functionals are lower than the one calculated with D1S, as it is expected from the lower surface energy values of BCP reported above. The smaller values of the fission barriers for the $\mathrm{BCP}$ functional results go in the right direction as compared to the experimental estimations $[12,13]$ but the effect of triaxiality in the first barrier in the case of the BCP functional remains to be studied. The impact in the spontaneous fission half-life $t_{\mathrm{SF}}$ of ${ }^{240} \mathrm{Pu}$ is also uncertain as this quantity not only depends on the topology of the PES but also on the collective inertia that has not been considered here. Preliminary calculations not including triaxiallity (therefore producing too high values for the half-life) yield $t_{\mathrm{SF}}=1.2 \times 10^{28} \mathrm{~s}$ for BCP1, $1.1 \times 10^{27} \mathrm{~s}$

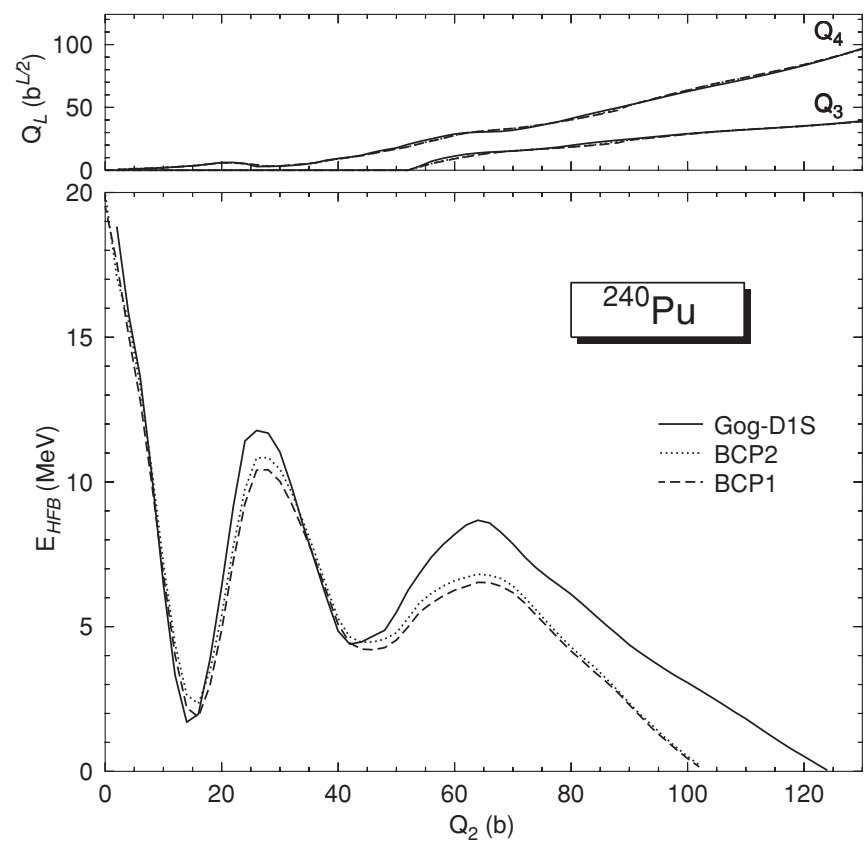

FIG. 5. Fission properties for the nucleus ${ }^{240} \mathrm{Pu}$ computed with the two parametrizations of the BCP functional as well as with the Gogny-D1S force. In the lower panel the potential energy surfaces are depicted as a function of the quadrupole moment and with respect to a reference energy of $-1810 \mathrm{MeV}$. In the upper panel the octupole and hexadecapole moments are presented.

for $\mathrm{BCP} 2$, and $1.5 \times 10^{26} \mathrm{~s}$ for D1S. Therefore it seems that the larger values of the collective inertia obtained for the BCP functionals (consistent with their lower pairing correlations) somehow counteract their lower fission barriers providing a longer half-life Although the shapes involved in fission are mainly characterized by its quadrupole moment, higher multipole deformations can be important for describing some fine details of the nuclear dynamics. In the top panel of Fig. 5 the expectation values of the octupole and hexadecapole moments are displayed as a function of $Q_{20}$ and striking similarity between the three results is observed.

The similarity between the results obtained with the $\mathrm{BCP}$ functionals and the Gogny force calls for a comparison of the underlying single particle structure that, as is well known, is essential in determining the response of the system to deformation. The most thorough comparison would involve the analysis of the single particle energy plots as a function of quadrupole deformation (Nilsson diagrams) but this is a demanding task that is deferred to a longer publication. We just want to mention here that the most relevant feature of the Nilsson diagrams, namely the shell gaps determining the location of minima are rather similar for both the BCP functionals and the Gogny force. This is so in spite of the different effective mass ratios $\mathrm{m}^{*} / \mathrm{m}$ in the two cases, one for the BCP functionals and 0.7 for Gogny-D1S, that imply a higher level density in the former case. To illustrate the different level densities we have plotted in Fig. 6 for the nucleus ${ }^{160}$ Dy the single particle levels at sphericity $\left(Q_{20}=0\right)$ for BCP1 and Gogny-D1S both for protons and neutrons. The higher level density of BCP's single particle levels is clearly 


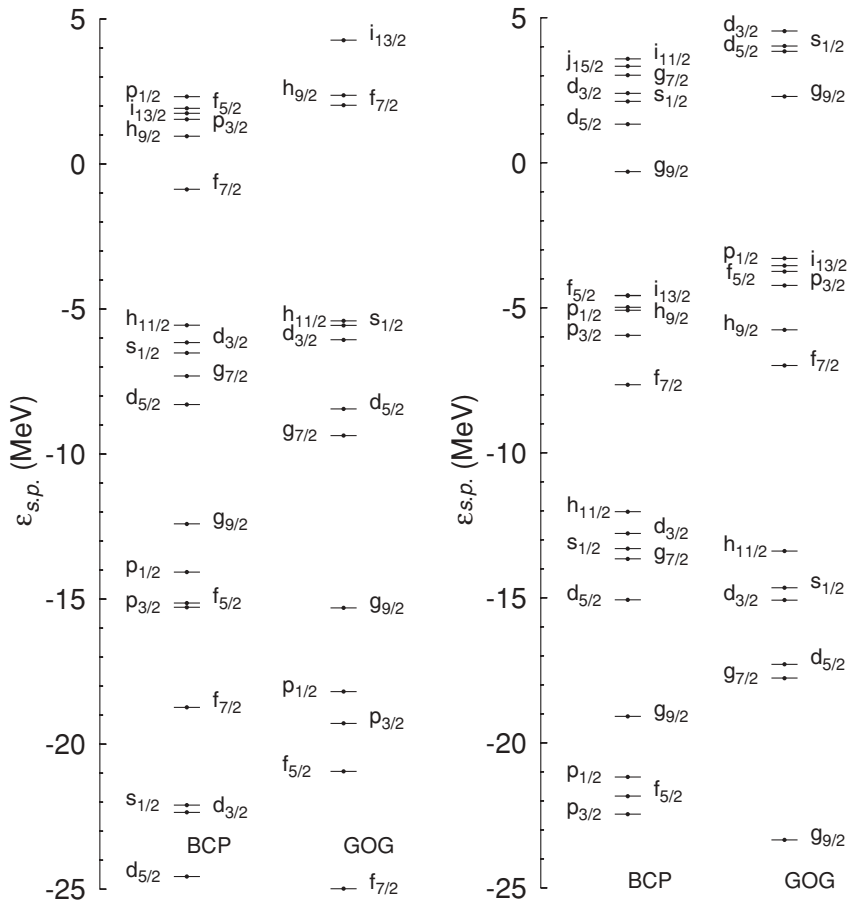

FIG. 6. Spherical single particle energies for protons (left panel) and neutrons (right panel) for the nucleus ${ }^{160} \mathrm{Dy}$. The results for the BCP1 functional and the Gogny force (GOG) are given. To help identify the levels their labels alternate their position with respect to the line assigned to them.

observed. Results for BCP2 are not included, since they are very similar to the ones of $\mathrm{BCP} 1$.
We have analyzed by means of a few selected examples the performance of the new BCP functional in what concerns quadrupole deformation. The tests performed are quite demanding as they include fission barriers for Pu or quadrupole properties over a wide range of isotopes in various regions of the nuclide chart. We have compared the results of our mean field calculations with experimental data whenever possible and found good agreement comparable to the one obtained for spherical magic or semimagic nuclei. Other quantities not directly related to experiment like the topology of the potential energy surfaces have been compared to the results of a well performing force and with long tradition in the field, namely the Gogny-D1S force and the results are comparable and of the same quality. Minor differences could be attributed to a slightly lower surface tension for BCP compared to D1S and to the different pairing interactions used. From these exploratory calculations we can conclude that the BCP functional with the $\mathrm{BCP} 1$ and $\mathrm{BCP} 2$ parametrization can be used with confidence in the study of nuclear properties related to deformation. Obviously a more thorough study of the BCP functional with respect to deformation has to be carried out as, e.g., the study of octupole deformation, triaxiallity, collective inertia, including the moment of inertia, etc. Work in this direction is underway.

This work has been partially supported by the CICyT-IN2P3 and CICyT-INFN collaborations. L.M.R. acknowledges financial support from the DGI of the MEC (Spain) under Project FIS2004-06697. X.V. acknowledges support from grants FIS2005-03142 of the MEC (Spain) and FEDER and 2005SGR-00343 of Generalitat de Catalunya.
[1] M. Baldo, P. Schuck, and X. Viñas, nucl-th/0706. 0658v2.

[2] M. Baldo, C. Maieron, P. Schuck, and X. Viñas, Nucl. Phys. A736, 241 (2004), and references therein.

[3] E. Garrido, P. Sarriguren, E. Moya de Guerra, and P. Schuck, Phys. Rev. C 60, 064312 (1999).

[4] J. F. Berger, M. Girod, and D. Gogny, Comput. Phys. Commun. 63, 365 (1991).

[5] J. F. Berger, M. Girod, and D. Gogny, Nucl. Phys. A502, 85c (1989).

[6] G. A. Lalazissis, J. König, and P. Ring, Phys. Rev. C 55, 540 (1997).
[7] E. Chabanat, P. Bonche, P. Haensel, J. Mayer, and R. Schaeffer, Nucl. Phys. A627, 710 (1997); A635, 231 (1998).

[8] P. Ring and P. Schuck, The Nuclear Many-Body Problem (Springer, Berlin, 1980).

[9] M. Farine (private communication).

[10] K. Rutz, M. Bender, P.-G. Reinhard, and J. A. Maruhn, Phys. Lett. B468, 1 (1999).

[11] M. Warda, J. L. Egido, L. M. Robledo, and K. Pomorski, Phys. Rev. C 66, 014310 (2002).

[12] R. B. Firestone, V. S. Sirley, S. Y. F. Chu, C. M. Baglin, and J. Zipkin, Table of Isotopes (John Wiley and Sons, New York, 1996).

[13] S. Bjornholm and J. E. Lynn, Rev. Mod. Phys. 52, 725 (1980). 